\title{
APPLICATIONS OF THE TRANSFER TO STABLE HOMOTOPY THEORY
}

\author{
BY DANIEL S. KAHN ${ }^{1}$ AND STEWART B. PRIDDY ${ }^{2}$
}

Communicated by E. H. Brown, Jr., May 18, 1972

In this note we outline the construction, properties and several applications of a transfer morphism for the generalized cohomology of finite coverings. Our principal application consists of a proof of a conjecture of M. E. Mahowald [9] and G. W. Whitehead [14]. Let $\lambda: \Sigma^{n} R P^{n-1} \rightarrow S^{n}$ be the adjoint of $R P^{n-1} \stackrel{i_{n}}{\longrightarrow} O_{n} \stackrel{j_{n}}{\longrightarrow} \Omega^{n} S^{n}$ where $i_{n}$ represents a line $L$ through the origin in $R^{n}$ as the reflection in the hyperplane perpendicular to $L$ and $j_{n}$ represents an element of $O_{n}$ as a map $\left(R^{n} \cup \infty, \infty\right) \rightarrow$ $\left(R^{n} \cup \infty, \infty\right)$. Then, for $0<i<n-1$,

$$
\lambda_{n+i}: \pi_{n+i}\left(\Sigma^{n} R P^{n-1}\right) \rightarrow \pi_{n+i}\left(S^{n}\right)
$$

is an epimorphism of 2-primary components (see §3).

The existence of the transfer seems to be well known [13], but we know of no published account. In $\$ 1$ we outline such a construction. The essential connection between the transfer and stable homotopy theory is provided by a stable map which yields the transfer as an induced homomorphism (Proposition 1.7). As an immediate consequence, the transfer commutes with stable cohomology operations. This generalizes the same result for ordinary cohomology of groups proved by Evens [7]; similarly it gives an alternate proof of Quillen's result [12] that the localized Adams operations $\psi^{p}\left[p^{-1}\right]$ commute with the transfer in $K$-theory. As a final application we given a stable decomposition of $\left(\Omega^{\infty} S^{\infty}\right)_{0}$. Details will appear elsewhere,

We would like to thank M. G. Barratt and M. E. Mahowald for many helpful conversations. Further thanks are due J. F. Adams for his critical comments on an earlier version of this work. In particular, he pointed out that our original argument could be modified so that the epimorphism (0.1) derives from a geometric splitting (see Theorem 3.1). A more detailed analysis of this point will appear in a forthcoming article by Adams. We have recently learned that F. W. Roush has also studied the transfer.

1. The transfer for generalized cohomology. Let $\pi: E \rightarrow B$ be a finite covering of degree $N$ (for the purposes of this note we assume $E$

\footnotetext{
AMS 1970 subject classifications. Primary 55B20, 55E45; Secondary 21J05; 55B15.

1 Partially supported by NSF grant GP-19815.

2 Partially supported by NSF grant GP-25335.
} 
connected). Let $\pi_{1}(E)=H, \pi_{1}(B)=G$; then we may replace $\pi: E \rightarrow B$ by the homeomorphic covering $p: X / H \rightarrow X / G$ where $X$ is the universal covering space of $B$ (acted upon freely on the right by $G$ ) and $p$ is the projection. Let $G / H=\left\{\tau_{1} H, \tau_{2} H, \ldots, \tau_{N} H\right\}$ be the left cosets of $H$ in $G$; then $G$ acts on the left of $G / H$ as a group of permutations, i.e., we have a representation $\rho: G \rightarrow \mathscr{S}_{N}\left(\mathscr{S}_{N}=\right.$ symmetric group on $\left.\{1, \ldots, N\}\right)$ defined by $g \tau_{i}=\tau_{\rho(g)(i)} h_{i}$ for $g \in G$ and uniquely determined $\rho(g) \in \mathscr{S}_{N}$ and $h_{i} \in H$.

Let $W G$ be a contractible $\mathrm{CW}$ complex on which $G$ acts freely on the left and similarly for $W \mathscr{S}_{N}$. Define

$$
\Phi: X \underset{G}{\times} W G \rightarrow(X / H)^{N} \underset{\mathscr{S}_{N}}{\times} W \mathscr{S}_{N}
$$

by $\Phi(x, \omega)=\left(\overline{x \tau_{1}}, \ldots, \overline{x \tau_{N}}, \rho_{*}(\omega)\right)$ where $G$ acts on the left of $X$ by $g \cdot x=x g^{-1}$ and $\mathscr{S}_{N}$ acts on the left of $(X / H)^{N}$ by permuting coordinates, i.e. $\sigma\left(x_{1}, \ldots, x_{N}\right)=\left(x_{\sigma^{-1}(1)}, \ldots, x_{\sigma^{-1}(N)}\right)$. The element $\overline{x \tau_{i}}$ is the class of $\overline{x \tau_{i}}$ in $X / H$ and $\rho: W G \rightarrow W \mathscr{S}_{N}$ is the induced map.

LEMMA 1.1. $\Phi$ is well defined and its homotopy class [Ф] is independent of the choice of coset representatives $\left\{\tau_{i}\right\}$.

Because of the evident homotopy equivalence $X / G \simeq X \times_{G} W G$ we shall often consider $\Phi$ as a map $\Phi: X / G \rightarrow(X / H)^{N} \times \mathscr{S}_{N} W \mathscr{S}_{N}$.

REMARK 1.2. Evens [8] has defined a group monomorphism $G \rightarrow$ $\mathscr{S}_{N} \int H$. It can be shown that the induced map $B G \rightarrow B\left(\mathscr{S}_{N} \int H\right)=$ $(B H)^{N} \times_{\mathscr{S}_{N}} W \mathscr{S}_{N}$ agrees up to homotopy with $\Phi$ for the covering $W G / H-W G / G .\left(\mathscr{S}_{N} \int H\right.$ is the wreath product [8].)

Let $(\cdot)^{+}$denote the addition of a disjoint point as base point.

Definition. 1.3. The pretransfer $T: X / G \rightarrow\left((X / H)^{+}\right)^{N} \times \mathscr{S}_{N} W \mathscr{S}_{N}$ for the $N$-fold covering $p: X / H \rightarrow X / G$ is the composite

$$
X / G \stackrel{\oplus}{\rightarrow}(X / H)^{N} \underset{\mathscr{S}_{N}}{\times} W \mathscr{S}_{N} \rightarrow\left((X / H)^{+}\right)^{N} \underset{\mathscr{S}_{N}}{\times} W \mathscr{S}_{N},
$$

where the second map is induced by the inclusion $(X / H) \rightarrow(X / H)^{+}$.

We now define the transfer morphism. Suppose $\mathscr{Y}$ is an $\Omega$-spectrum. (All $\Omega$-spectra and morphisms of such are assumed strict, i.e. $\mathscr{Y}_{i}=\Omega \mathscr{Y}_{i+1}$, etc. May [10] shows that these spectra are adequate.) Recall that there are Dyer-Lashof maps [4]

$$
\theta_{q}^{N}(\mathscr{Y})=\theta_{q}^{N}: \mathscr{Y}_{q}^{N} \underset{\mathscr{S}_{N}}{\times} W \mathscr{S}_{N} \rightarrow \mathscr{Y}_{q}
$$

defined using the iterated loop space structure of $\mathscr{Y}_{q}$.

DEFINITION 1.4. The (Y)-cohomology) transfer morphism, for the $N$-fold covering $p: X / H \rightarrow X / G, p^{!}: H^{q}(X / H ; \mathscr{Y}) \rightarrow H^{q}(X / G ; \mathscr{Y})$ is defined by $p^{!}(\alpha)=\left[\theta_{q}^{N} \circ\left(a^{N} \times_{\mathscr{S}_{N}} 1\right) \circ T\right]$, 


$$
(X / G) \stackrel{T}{\rightarrow}\left((X / H)^{+}\right)^{N} \underset{\mathscr{S}_{N}}{\times} W \mathscr{S}_{N} \stackrel{a^{N} \times \mathscr{S}_{N}}{\longrightarrow}\left(\mathscr{Y}_{q}\right)^{N} \underset{\mathscr{S}}{\times} W \mathscr{S}_{N} \stackrel{\theta_{q}^{N}}{\rightarrow} \mathscr{Y}_{q}
$$

where $a:(X / H)^{+} \rightarrow \mathscr{Y}_{q}$ represents $\alpha \in H^{q}(X / H ; \mathscr{Y})$.

Proposition 1.5. $p^{\prime}$ is well defined and natural with respect to morphisms of covering maps and morphisms of $\Omega$-spectra. Moreover, $p !$ commutes with compositions of coverings.

1.6. Set $Q\left((X / H)^{+}\right)=\bigcup_{n} \Omega^{n} \Sigma^{n}\left((X / H)^{+}\right)$. Let $f:(X / G)^{+} \rightarrow Q\left((X / H)^{+}\right)$ be the pointed extension to $(X / G)^{+}$of $\theta_{q}^{N} \circ\left(i^{N} \times_{\mathscr{S}_{N}} 1\right) \circ T$ (see Definition 1.4), where $i:(X / H)^{+} \rightarrow Q\left((X / H)^{+}\right)$is the inclusion, i.e. $[f]=p^{!}([i]) \in$ $H^{0}(X / G ; \mathscr{Y})$ for the $\Omega$-spectrum $\mathscr{Y}$ with $\mathscr{Y}_{k}=Q\left(\Sigma^{k}\left((X / H)^{+}\right)\right)$. (Y is the $\Omega$-spectrum associated to the suspension spectrum of $(X / H)^{+}$.) If $X$ is l-dimensional, $f$ is homotopic to a map $\hat{f}:(X / G)^{+} \rightarrow \Omega^{l+1} \Sigma^{l+1}\left((X / H)^{+}\right) \subset$ $Q\left((X / H)^{+}\right)$. Thus $(\operatorname{adj} \hat{f}): \Sigma^{l+1}\left((X / G)^{+}\right) \rightarrow \Sigma^{l+1}\left((X / H)^{+}\right)$. With this notation we state

Proposition 1.7. If $X / H$ has finite dimension $l$ and $\mathscr{E}$ is an $\Omega$-spectrum, then

$$
\begin{gathered}
H^{*}(X / H ; \mathscr{E}) \longrightarrow H^{*}(X / G ; \mathscr{E}) \\
\approx \uparrow \sigma \quad \approx \uparrow \sigma \\
H^{*+l+1}\left(\Sigma^{l+1}\left((X / H)^{+}\right) ; \mathscr{E}\right) \underset{(\operatorname{adj} \hat{f})^{*}}{\longrightarrow} H^{*+l+1}\left(\Sigma^{l+1}\left((X / G)^{+}\right) ; \mathscr{E}\right)
\end{gathered}
$$

commutes, where $\sigma$ denotes the suspension isomorphism.

COROLlaRY 1.8. $p$ ! is a homomorphism and commutes with stable cohomology operations for finite complexes.

2. Agreement with other definitions of the transfer. Let $t^{*}: H^{*}(X / H ; A) \rightarrow$ $H^{*}(X / G ; A)$ denote the ordinary cohomology transfer [6] for the $N$-fold covering $p: X / H \rightarrow X / G$ where $A$ is an abelian group.

Proposition 2.1. Let $\mathscr{K}(A)$ denote the Eilenberg-Mac Lane spectrum. Then

$$
t^{*}=p^{!}: H^{*}(X / H ; \mathscr{K}(A)) \rightarrow H^{*}(X / G ; \mathscr{K}(A)),
$$

i.e. the transfer $p$ ! agrees with the classical transfer for ordinary cohomology theory.

Remark 2.2. Consider the situation of Proposition 1.7. It follows from the universal coefficient theorem that the induced ordinary homology homomorphism $(\operatorname{adj} \hat{f})_{*}$ with $Z_{p}$ coefficients $(p$ a prime) is equivalent to the classical ordinary homology transfer. This fact can be proved directly and holds also for the case of integer coefficients. 
REMARK 2.3. In the situation of Proposition 1.7, consider the map

$$
\begin{aligned}
(\operatorname{adj} \hat{f}): \Sigma^{l+1}\left((X / G)^{+}\right) & =\Sigma^{l+1}(X / G) \vee S^{l+1} \rightarrow \Sigma^{l+1}(X / H) \vee S^{l+1} \\
& =\Sigma^{l+1}\left((X / H)^{+}\right) .
\end{aligned}
$$

Since $\Sigma^{l+1}(X / H)$ is $(l+1)$-connected, one may pinch to a point the two $(l+1)$-spheres and obtain a map $\Sigma^{l+1}(X / G) \rightarrow \Sigma^{l+1}(X / H)$ whose induced homology homomorphism is equivalent to the transfer on reduced homology groups.

To obtain similar results for $K$-theory, one observes that Atiyah's definition [1] of the transfer bundle $\rho_{*}(E): X / G \rightarrow B U_{N k}$ of a bundle $E: X / H \rightarrow B U_{k}$ is given by

$X / G \stackrel{E}{\rightarrow}(X / H)^{N} \underset{\mathscr{S}_{N}}{\times} W \mathscr{S}_{N} \stackrel{E^{N} \times \mathscr{S}_{N} 1}{\longrightarrow}\left(B U_{k}\right)^{N} \underset{\mathscr{S}_{N}}{\times} W \mathscr{S}_{N}=B\left(\mathscr{S}_{N} \int U_{k}\right) \rightarrow B U_{N k^{*}}$

Proposition 2.4. Let $\mathscr{K}$ denote either the BU or BO spectrum. Then

$$
\rho_{*}=p^{!}: H^{*}(X / H ; \mathscr{K}) \rightarrow H^{*}(X / G ; \mathscr{K}),
$$

i.e. the transfer $p$ ! agrees with Atiyah's transfer for K-theory.

The proof relies on the homotopy commutative diagram

$$
\begin{aligned}
& \left(B U_{k}\right)^{N} \times W \mathscr{S}_{N}=B\left(\mathscr{S}_{N} \int U_{k}\right) \rightarrow B U_{k N} \\
& (B U)^{N} \underset{\mathscr{S}_{N}}{\times} W \mathscr{S}_{N} \longrightarrow B U
\end{aligned}
$$

of Boardman (unpublished). Similarly for $U$ replaced by $O$.

3. Applications. (i) Let $[m]$ denote the base point of $\left(Q S^{0}\right)_{m}$, the path component of a map $S^{n} \rightarrow S^{n}$ of degree $m$, and let $\gamma_{m}: B \mathscr{S}_{m} \rightarrow\left(Q S^{0}\right)_{0}$ be the composite map

$$
B \mathscr{S}_{m}=[1]^{m} \underset{\mathscr{S}_{m}}{\times} W \mathscr{S}_{m} \rightarrow\left(\left(Q S^{0}\right)_{1}\right)^{m} \underset{\mathscr{S}_{m}}{\times} W \mathscr{S}_{m} \stackrel{\theta^{m}}{\longrightarrow}\left(Q S^{0}\right)_{m} \stackrel{*[-m]}{\longrightarrow}\left(Q S^{0}\right)_{0}
$$

where $\theta^{m}$ is the Dyer-Lashof map and * is the loop product for $Q S^{0}$. We shall equate $B \mathscr{S}_{2}=R P^{\infty}$ and write $\gamma=\gamma_{2}: R P^{\infty} \rightarrow\left(Q S^{0}\right)_{0}, \gamma$ has a canonical extension $\bar{\gamma}: Q\left(R P^{\infty}\right) \rightarrow\left(Q S^{0}\right)_{0}$ (see below). Similarly for any map $\phi: R P^{\infty} \rightarrow\left(Q S^{0}\right)_{0}$.

THEOREM 3.1. Let $\phi: R P^{\infty} \rightarrow\left(Q S^{0}\right)_{0}$ be a map such that $\phi_{*}\left(e_{1}\right)=\gamma_{*}\left(e_{1}\right)$ where $e_{1}$ is the generator of $H_{1}\left(R P^{\infty} ; Z_{2}\right)$. Then there exists a map $\psi:\left(Q S^{0}\right)_{0} \rightarrow Q\left(R P^{\infty}\right)$ such that $\bar{\phi} \circ \psi$ is a mod 2 equivalence, i.e. $\bar{\phi}$ splits $\bmod 2$.

REMARK 3.2. Any $\phi: R P^{\infty} \rightarrow\left(Q S^{0}\right)_{0}$ which is nontrivial on the fundamental group will do. In particular the map $\lambda$ of the introduction is 
such a $\phi$. It is an immediate consequence of Theorem 3.1 that the homomorphism (0.1) is surjective.

Before sketching a proof of Theorem 3.1, we recall that there is a natural transformation $h: Q Q(\cdot) \rightarrow Q(\cdot)$ such that, if $Y$ is an infinite loop space with retraction map $r: Q Y \rightarrow Y\left[10\right.$, p. 451], then $r \circ Q(r)=r \circ h_{Y}$. (If $\phi: X \rightarrow Y, \bar{\phi}=r \circ Q(\phi): Q X \rightarrow Y$ is the canonical extension mentioned above.) We fix the following notation: $\hat{Q}_{m}(\cdot)=(\cdot)^{m} \times \mathscr{S}_{m} W \mathscr{S}_{m}$ and $\mathscr{S}\left(2^{k}, 2\right)$ is a 2-Sylow subgroup $\mathscr{S}_{2} \int \cdots \int \mathscr{S}_{2}$ ( $k$-fold wreath product) of $\mathscr{S}_{2^{k}}$. We have $B \mathscr{S}\left(2^{k}, 2\right)=\hat{Q}_{2} \ldots Q_{2} B \mathscr{S}_{2}\left(k-1 \hat{Q}_{2}\right.$ 's). Recall that the index $m=\left[\mathscr{S}\left(2^{k}, 2\right): \mathscr{S}_{2^{k}}\right]$ is odd.

SKETCH OF PROOF OF THEOREM 3.1. Consider the following two composite maps:

$$
\begin{aligned}
\Sigma^{\infty} B \mathscr{S}_{2^{k}} \stackrel{f_{1}}{\longrightarrow} \Sigma^{\infty} B \mathscr{S}\left(2^{k}, 2\right) \stackrel{f_{2}}{\longrightarrow} \Sigma^{\infty} B \mathscr{S}_{2^{k}} \stackrel{f_{3}}{\longrightarrow} \Sigma^{\infty}\left(Q S^{0}\right)_{0}, \\
\Sigma^{\infty} B \mathscr{S}_{2^{k}} \stackrel{f_{1}}{\longrightarrow} \Sigma^{\infty} B \mathscr{S}\left(2^{k}, 2\right)=\Sigma^{\infty} \hat{Q}_{2} \cdots \hat{Q}_{2} B \mathscr{S}_{2} \stackrel{g_{2}}{\longrightarrow} \Sigma^{\infty} \hat{Q}_{2^{k-1}} B \mathscr{S}_{2} \\
\stackrel{g_{3}}{\longrightarrow} \Sigma^{\infty} Q B \mathscr{S}_{2} \stackrel{g_{4}}{\longrightarrow} \Sigma^{\infty}\left(Q S^{0}\right)_{0}
\end{aligned}
$$

and set $F=f_{3} f_{2} f_{1}, G_{\phi}=g_{4} g_{3} g_{2} f_{1}$.

$\Sigma^{\infty}$ denotes the suspension spectrum functor. $f_{1}$ is the map of Remark 2.3 associated to the covering $B \mathscr{S}\left(2^{k}, 2\right) \rightarrow B \mathscr{S}_{2^{k}} ; f_{2}$ is induced by the inclusion $\mathscr{S}\left(2^{k}, 2\right) \subset S_{2 k} ; f_{3}=\Sigma^{\infty} \gamma_{2 k} ; g_{2}$ is induced by the wreath product; $g_{3}$ a Dyer-Lashof map for $Q B \mathscr{S}_{2}$; and $g_{4}$ is $\Sigma^{\infty}(\bar{\phi})$. With the exception of $f_{1}$, each of these is $\Sigma^{\infty}$ of a map.

$$
\left(f_{2} f_{1}\right)_{*}: H_{*}\left(\Sigma^{\infty} B \mathscr{S}_{2^{k}} ; Z_{2}\right) \rightarrow H_{*}\left(\Sigma^{\infty} B \mathscr{S}_{2^{k}} ; Z_{2}\right)
$$

is the identity since the ordinary homology transfer followed by inclusion is multiplication by the index, which in this case is odd. Since $\gamma_{2^{k} *}$ is a mod 2 homology isomorphism in dimensions $\leqq 2^{k-1}$ [3], [11], $F_{*}$ is a mod 2 homology isomorphism in dimensions $\leqq 2^{k-1}$. An augmentation type filtration argument comparing $F_{*}$ to $G_{\gamma *}$ shows that $G_{\gamma *}$ is also a mod 2 isomorphism in dimensions $\leqq 2^{k-1}$. Then a length type filtration argument comparing $G_{\gamma *}$ to $G_{\phi *}$ shows that $G_{\phi *}$ is a mod 2 isomorphism in dimensions $\leqq 2^{k-1}$.

Apply $\Omega^{\infty}$, restrict to 2-primary factors and obtain a commutative diagram

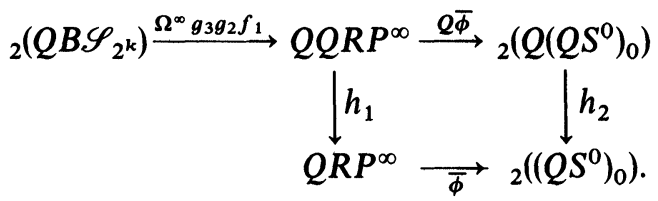

$(Q \bar{\phi}) \circ\left(\Omega^{\infty} g_{3} g_{2} f_{1}\right)$ is a mod 2 equivalence in dimensions $\leqq 2^{k-1}$. An inverse limit argument (using finiteness of all homotopy groups in sight) 
provides a map $k:{ }_{2}\left(Q\left(Q S^{0}\right)\right) \rightarrow Q Q R P^{\infty}$ such that $(Q \bar{\phi}) \circ k$ is homotopic to the identity map. Let $i:{ }_{2}\left(Q S^{0}\right)_{0} \rightarrow{ }_{2} Q\left(Q S^{0}\right)_{0}$ be the canonical section of $h_{2}$. Then $\psi=h_{1} \circ k \circ i$ satisfies Theorem 3.1 on 2-primary factors. Theorem 3.1 now follows.

REMARK 3.5. An analogous result holds for odd primes using $p\left(B \mathscr{S}_{p}\right)$ in place of $B \mathscr{S}_{2}$. Let $\phi:{ }_{p}\left(B \mathscr{S}_{p}\right) \rightarrow\left(Q S^{0}\right)_{0}$ be any map such that $\phi_{*}$ is nontrivial on $H_{2 p-3}\left(; Z_{p}\right)$. Then $\bar{\phi}: Q\left({ }_{p}\left(B \mathscr{S}_{p}\right)\right) \rightarrow\left(Q S^{0}\right)_{0}$ splits mod $p$.

(ii) In [5], Dold gives an elegant proof of Nakaoka's decomposition of the homology groups oj $B \mathscr{S}_{n}$. Using Proposition 1.7, one can geometrically mimic Dold's proof obtaining

Proposition 3.6. For $2 \leqq n \leqq \infty$, there is an equivalence of spectra

$$
\Sigma^{\infty}\left(B \mathscr{S}_{n}\right) \rightarrow \bigvee_{k=2}^{n} \Sigma^{\infty}\left(B \mathscr{S}_{k} / B \mathscr{S}_{k-1}\right) .
$$

Proposition 3.7. For $2 \leqq n \leqq \infty$, there is a homotopy equivalence

$$
Q\left(B \mathscr{S}_{n}\right) \rightarrow \prod_{k=2}^{n} Q\left(B \mathscr{S}_{k} / B \mathscr{S}_{k-1}\right) .
$$

We observe that the homology equivalence $B \mathscr{S}_{\infty} \rightarrow\left(Q S^{0}\right)_{0}$ [3] implies the corresponding decompositions for $\Sigma^{\infty}\left(Q S^{0}\right)_{0}$ and $Q\left(Q S^{0}\right)_{0}$. (Compare [2].)

\section{REFERENCES}

1. M. Atiyah, Characters and cohomology of finite groups, Inst. Hautes Ètudes Sci. Publ. Math. No. 9 (1961), 23-64. MR 26 \#6228.

2. M. Barratt and P. J. Eccles, On a theorem of D. S. Kahn's, University of Manchester (mimeograph).

3. M. Barratt, D. Kahn and S. Priddy, On $\Omega^{\infty} S^{\infty}$ and the infinite symmetric group, Proc. Sympos. Pure Math., vol. 22, Amer. Math. Soc., Providence, R.I., 1971.

4. E. Dyer and R. Lashof, Homology of iterated loop spaces, Amer. J. Math. 84 (1962), 35-88. MR 25 \# 4523.

5. A. Dold, Decomposition theorems for S(n)-complexes, Ann. of Math. (2) 75 (1962), 8-16. MR 25 \# 569.

6. B. Eckmann, On complexes with operators, Proc. Nat. Acad. Sci. U.S.A. 39 (1953), 35-42. MR 15, 459.

7. L. Evens, Steenrod operations and transfer, Proc. Amer. Math. Soc. 19 (1968), 13871388. MR 38 \# 1669.

8. - A generalization of the transfer map in the cohomology of groups, Trans. Amer. Math. Soc. 108 (1963), 54-65. MR 27 \#3686.

9. M. Mahowald, The metastable homotopy of $S^{n}$, Amer. Math. Soc. Memoir No. 72 (1967). MR 38 \# 5216.

10. J. P. May, Categories of spectra and infinite loop spaces, Category Theory, Homology Theory and their Applications, (Battelle Institute Conference, Seattle, Wash., 1968), vol. III, Springer, Berlin, 1969, pp. 448-479. MR 40 \#2073.

11. M. Nakaoka, Decomposition theorem for homology groups of symmetric groups, Ann. of Math. (2) 71 (1960), 16-42. MR $22 \# 2989$.

12. D. Quillen, The Adams conjecture, Topology 10 (1970), 67-80. MR 43 \#5525. 
13. G. Segal, Homotopy-everything $H$-spaces (preprint).

14. G. Whitehead, Recent advances in homotopy theory, Regional Conference Series in Math., no. 5, Amer. Math. Soc., Providence, R.I., 1970.

Department of Mathematics, NorthWESTERn University, Evanston, IllinOIs 60201 